\title{
Recurrent Childhood Visual Pathway Astrocytoma
}

National Cancer Institute

\section{Source}

National Cancer Institute. Recurrent Childhood Visual Pathway Astrocytoma. NCI

Thesaurus. Code C7530.

The reemergence of visual pathway astrocytoma in childhood after a period of remission. 